Nature Reviews Genetics | AOP, published online 9 April 2015; doi:10.1038/nrg3939

\title{
ane
}

\section{Detailed probing of RNA structure in vivo}

Folding of RNAs into structural motifs is important for the function and regulation of both proteincoding and non-coding RNAs. Various methods have been devised to probe RNA structure on a global scale, and a particular drive is to move from studying RNAs that have been refolded in vitro to characterizing RNAs in their native cellular environments to account for their interactions with other cellular components such as RNA-binding proteins (RBPs). Two recent studies describe new methods to probe RNA structure in cells and provide new insights into gene regulation.

Experimental methods for characterizing RNA structure transcriptome-wide in cells typically use cell-permeable reagents that differentially react with RNA nucleotides depending on whether they are engaged in base-pairing interactions, followed by high-throughput sequencing as a readout for sites modified by the reagents. One challenge has been to develop reagents that reliably report structures of all four RNA nucleotides. Spitale et al. built on their previous work with 2-methylnicotinic acid imidazolide (NAI) - which reacts with the free hydroxyl group in the ribose of all four RNA nucleotides when they are unstructured - and showed that adding an azide group allowed the reagent to be biotin-labelled by click chemistry, which enabled selective purification of modified RNA and accurate reading of the RNA structure profile by sequencing.

Using this in vivo click selective 2 '-hydroxyl acylation and profiling experiment (icSHAPE), they profiled the structures of mRNAs globally inside mouse embryonic stem cells and also mRNAs extracted from these cells that were refolded in vitro. For translational start sites and ribosomal pause sites, the in vitro and in vivo RNA structures were highly similar, indicating that the structures of these translational control elements are intrinsically programmed into the RNA sequence. By contrast, some sequences containing binding motifs for RBPs underwent structural rearrangements in vivo, suggesting that RNA structure or accessibility was being modulated by RBP binding. Indeed, binding sites of the RBFOX2 and HuR RBPs could be predicted by icSHAPE data, as validated by direct immunoprecipitation and sequencing of RNAs bound by these RBPs using the individual nucleotide crosslinking immunoprecipitation (iCLIP) method.

Finally, Spitale et al. showed that the physiological RNA modification $N^{6}$-methyladenosine $\left(\mathrm{m}^{6} \mathrm{~A}\right)$ is associated with a characteristic RNA structure, and they used cells deficient for the key $\mathrm{m}^{6} \mathrm{~A}$ methyltransferase METTL3 to demonstrate that the structure is probably caused by the $\mathrm{m}^{6} \mathrm{~A}$ modification rather than being a favoured substrate for METTL3-mediated methylation.

In a separate study, Sugimoto et al. studied RBP-bound RNA structures by modifying the iCLIP procedure to incorporate an adaptor ligation step. This advance, named RNA hybrid and iCLIP (hiCLIP), allows particular RBPs to be isolated with their bound intramolecular and intermolecular RNA duplexes - rather than just the single RNA strands isolated by iCLIP - and then uses highthroughput sequencing to identify the constituent RNA hybrids.
Applying hiCLIP to the Staufen 1 (STAU1) RBP in human 293T cells, a notable result was the high prevalence of long-range RNA duplexes - that is, intramolecular interactions between regions that are hundreds of nucleotides apart in the primary RNA sequence and which are challenging to detect by most experimental or computational methods for probing RNA structure. These interactions included regions in the $3^{\prime}$ untranslated region ( $\left.3^{\prime} \mathrm{UTR}\right)$ just downstream of the stop codon hybridizing further downstream near the poly(A) site or upstream near the start codon.

Functional analyses identified roles for the long-range RNA structures in translation efficiency and RNA stability, as well as cytoplasmic splicing of XBP1 mRNA. Furthermore, decreased human genetic variation was found in the STAU1-bound duplexes in 3'UTRs compared with neighbouring regions, which might mean that disease-associated genetic variants in these regions act, in part, through disruption of these RNA structures.

Both studies thus contribute methods for dissecting how cells use and remodel structured RNA for gene regulation in cells. Future application to additional cell types, genetic backgrounds, stimuli and RBPs holds promise for further global insights into the functions and dynamics of RNA structure.

Darren J. Burgess

ORIGINAL RESEARCH PAPERS Spitale. R. C. et al. Structural imprints in vivo decode RNA regulatory mechanisms. Nature 519, 486-490 (2015) | Sugimoto, Y. et al. hiCLIP reveals the in vivo atlas of mRNA secondary structures recognized by Staufen 1. Nature 519, 491-494 (2015) 